\title{
Side-Communication Yields Efficiency of Ascending Auctions: The Two-Items Case
}

\author{
Ron $\mathrm{Lavi}^{1, \star}$ and Sigal Oren ${ }^{2, \star \star}$ \\ 1 Faculty of Industrial Engineering and Management \\ Technion - Israel Institute of Technology \\ ronlavi@ie.technion.ac.il \\ 2 Computer Science Department \\ Cornell University \\ sigal@cs.cornell.edu
}

\begin{abstract}
We analyze an ascending auction with anonymous itemprices, when there are two items that are substitutes. This popular auction format entails increased opportunities for bidders to coordinate bids, as the bidding process is longer, and since bidders see the other bids and can respond to various signaling. This has happened in many real auctions, e.g., in the Netherlands 3G Telecom Auction and in the FCC auctions in the US.

While on the face of it, such bidding behavior seems to harm economic efficiency, we show that side-communication may actually improve the social efficiency of the auction: We describe an ex-post sub-game perfect equilibrium, that uses limited side-communication, and is ex-post efficient. In contrast, without side-communication, we show that there is no ex-post equilibrium which is ex-post efficient in the ascending auction.

In the equilibrium strategy we suggest, bidders start by reporting their true demands at the first stages of the auction, and then perform a single demand reduction at a certain concrete point, determined using a single message exchanged between the bidders. We show that this limited signaling opportunity resolves the strategic problems of myopic bidding, and, quite surprisingly, improves social welfare instead of harming it.
\end{abstract}

Keywords: ascending auctions, myopic bidding, signaling, ex-post efficiency.

* Supported in part by grants from the Israeli Science Foundation, the Bi-national Science Foundation, the Israeli ministry of science, and by the Google Inter-university center for Electronic Markets and Auctions.

** Work done while this author was at the Technion, supported by grants from the Israeli Science Foundation and the Bi-national Science Foundation.

P. Coles et al. (Eds.): AMMA 2011, LNICST 80, p. 55, 2012.

(C) Institute for Computer Sciences, Social Informatics and Telecommunications Engineering 2012 